\title{
Not Every Number is the Sum or Difference of Two Prime Powers
}

\author{
By Fred Cohen and J. L. Selfridge
}

\begin{abstract}
Every odd number less than 262144 is the sum or difference of a power of two and a prime. An interesting example is $113921=p-2^{141}$. Using covering congruences, we exhibit a 26-digit odd number which is neither the sum nor difference of a power of two and a prime. The method is then modified to exhibit an arithmetic progression of numbers which are not the sum or difference of two prime powers.
\end{abstract}

In 1950, P. Erdös [1] used covering congruences to exhibit numbers not of the form $2^{n}+p$. Using similar methods, we study the sequences $2^{n}+M$ and $\pm\left(2^{n}-M\right)$ for a fixed integer $M$. In particular, we prove

THEOREM 1. There exists an arithmetic progression of odd numbers which are neither the sum nor difference of a power of two and a prime.

Via the proof of Theorem 1 and primality testing programs of M. Wunderlich, we show

COROLLARY. 47867742232066880047611079 is prime and neither the sum nor difference of a power of two and a prime.

A modification of the proof of Theorem 1 yields

THEOREM 2. There exist odd numbers which are neither the sum nor difference of a power of two and a prime power.

Curiously, numbers satisfying Theorem 1 appear relatively difficult to find. We have calculated (on an IBM 360) that if $M<2^{18}$ and $M$ is odd, then $M$ is the sum or difference of a power of two and a prime. The $M$ for which it is most difficult to verify this fact is 113921 . Here $113921+2^{141}$ is prime (proved using Lucas sequences) and 141 is the smallest $n$ such that $\left|113921 \pm 2^{n}\right|$ is prime.

Proof of Theorem 1 and its Corollary. Recall that a collection of congruences $n \equiv b_{i}\left(h_{i}\right)$ is called a covering if each integer satisfies at least one of the congruences. We exhibit two coverings of the integers. For each congruence in either cover, we require $M$ to satisfy a related congruence. Any $M$ which satisfies these related congruences enjoys the following property: There is a list of 18 primes such that for any nonnegative integer $n, M+2^{n} \equiv 0\left(p_{i}\right)$ and $M-2^{n} \equiv 0\left(p_{j}\right)$ for some $p_{i}$ and some $p_{j}$ in our list. Theorem 1 follows directly; the number in the corollary satisfies the requisite congruences on $M$.

Received May 23, 1974.

AMS (MOS) subject classifications (1970). Primary 10J15; Secondary 10-04.

Copyright $\odot 1975$, American Mathematical Society 
Congruences on $M$

$$
\begin{aligned}
& M+2^{n} \equiv 0\left(p_{i}\right) \\
& M+2^{1} \equiv 0(3) \\
& M+2^{0} \equiv 0(5) \\
& M+2^{6} \equiv 0(17) \\
& M+2^{10} \equiv 0(13) \\
& M+2^{2} \equiv 0(97) \\
& M+2^{10} \equiv 0(257) \\
& M+2^{18} \equiv 0(241) \\
& M \equiv 2^{n}\left(p_{i}\right) \\
& M \equiv 2^{0}(3) \\
& M \equiv 2^{0}(7) \\
& M \equiv 2^{17}(109) \\
& M \equiv 2^{35}(37) \\
& M \equiv 2^{11}(19) \\
& M \equiv 2^{5}(73) \\
& M \equiv 2^{25}(331) \\
& M \equiv 2^{1}(41) \\
& M \equiv 2^{31}(61) \\
& M \equiv 2^{2}(31) \\
& M \equiv 2^{3}(11) \\
& M \equiv 2^{4}(151)
\end{aligned}
$$

Covering Congruences

iff

$$
\begin{aligned}
n & \equiv b_{i}\left(h_{i}\right) \\
n & \equiv 1(2) \\
n & \equiv 0(4) \\
n & \equiv 6(8) \\
n & \equiv 10(12) \\
n & \equiv 2(48) \\
n & \equiv 10(16) \\
n & \equiv 18(24) \\
n & \equiv b_{i}\left(h_{i}\right) \\
n & \equiv 0(2) \\
n & \equiv 0(3) \\
n & \equiv 17(36) \\
n & \equiv 35(36) \\
n & \equiv 11(18) \\
n & \equiv 5(9) \\
n & \equiv 25(30) \\
n & \equiv 1(20) \\
n & \equiv 31(60) \\
n & \equiv 2(5) \\
n & \equiv 3(10) \\
n & \equiv 4(15) .
\end{aligned}
$$

iff

End Theorem 1 proof.

We remark that it is impossible to cover both $M+2^{n}$ and $M-2^{n}$ with primes less than 331. More precisely, for any $M$ there is an $n$ such that either $M+2^{n}$ or $M-2^{n}$ has all its prime factors greater than 330 . On the other hand, by using more primes we can dispense with 3 ; that is, we can find an $M$ and a set of 42 primes so that $3 M+2^{n}$ and $3 M-2^{n}$ are simultaneously covered.

Proof of Theorem 2. We find additional conditions on $M$ and additional primes (16 in all).

First we will insure that all terms, $M+2^{n}$, divisible by $3^{3}$ are divisible by 37 or 109. Put $M+2^{17} \equiv 0\left(3^{3}\right)$ and thus $3^{3}$ divides $M+2^{n}$ when $n \equiv 17(18)$. To cover $n \equiv 17(36)$ we put $M+2^{17} \equiv 0(37)$ and to cover $n \equiv 35(36)$ we put $M+$ $2^{35} \equiv 0(109)$.

Next we add conditions on the remaining primes covering $M+2^{n}$.

$$
\begin{array}{ll}
M+2^{8} \equiv 0\left(5^{2} \cdot 11\right) & M+2^{34} \equiv 0\left(97^{2} \cdot 389\right) \\
M+2^{6} \equiv 0\left(17^{2} \cdot 137\right) & M+2^{18} \equiv 0\left(241^{2} \cdot 1447\right) \\
M+2^{2} \equiv 0\left(13^{2} \cdot 53\right) & M+2^{10} \equiv 0(257 \cdot 673)
\end{array}
$$

Note that we have used our cover from Theorem 1 with the positions of 13 and 97 interchanged. 
Now notice that $M+2^{n}$ can never be a prime power.

Consider $\left|M-2^{n}\right|$. First we have $M \equiv 2^{8}\left(3^{2}\right)$ from above, and each term with $n \equiv 8(6)$ is either $n \equiv 8(12)$ or $n \equiv 2(4)$. But since $M \equiv 2^{8}(13)$ and $M \equiv 2^{2}(5)$ from above, we have a second prime factor whenever $3^{2}$ divides $\left|M-2^{n}\right|$. Finally, we add conditions for the remaining primes covering $\left|M-2^{n}\right|$, noticing that the conditions for 11,37 , and 109 are consistent with those already described.

$$
\begin{aligned}
M & \equiv 2^{3}\left(7^{2} \cdot 43\right) \quad\left(\text { note } M \equiv 2^{0}(3 \cdot 7)\right) \\
M & \equiv 2^{17}\left(109^{2} \cdot 2617\right) \\
M & \equiv 2^{35}\left(37^{2} \cdot 149\right) \\
M & \equiv 2^{11}\left(19^{2} \cdot 571\right) \\
M & \equiv 2^{5}\left(73^{2} \cdot 439\right) \\
M & \equiv 2^{25}\left(331^{2} \cdot 1987\right) \\
M & \equiv 2^{1}\left(41^{2} \cdot 83\right) \\
M & \equiv 2^{31}(61 \cdot 1321) \\
M & \equiv 2^{2}\left(31^{2} \cdot 311\right) \\
M & \equiv 2^{3}\left(11^{2} \cdot 23\right) \\
M & \equiv 2^{19}\left(151^{2} \cdot 907\right) \quad\left(\text { note } M \equiv 2^{4}(3 \cdot 151)\right)
\end{aligned}
$$

Hence $\left|M-2^{n}\right|$ has at least two distinct prime factors provided that it is greater than 331. End Theorem 2 proof.

A routine use of Lehmer's linear equation solver shows that 61206699060672 767780921156017566254819576161631922981734368549334512406741 74209468558999326569 satisfies these congruences.

Finally, we wish to thank M. Wunderlich for his primality testing program and D. H. Lehmer for his multiple-precision package.

Mathematics Department

Northern Illinois University

DeKalb, Illinois 60115

1. P. ERDÖS, "On integers of the form $2^{k}+p$ and some related problems," Summa Brasil. Math., v. 2, 1950, pp. 113-123. MR 13, 437. 